\title{
Le bon usage du médicament chez le sujet âgé de 80 ans et plus
}

\author{
Thierry Marquet, ${ }^{1}$ Catherine Deguines, ${ }^{2}$ Yves Juillet ${ }^{3}$ et les participants à la Table Ronde \\ $n^{\circ} 5$ de Giens XX* \\ 1 Laboratoires Lundbeck SAS, Paris, France \\ 2 Agence française de sécurité sanitaire des produits de santé (Afssaps), Saint-Denis, France \\ 3 Les Entreprises du Médicament, Paris, France
}

Résumé

\begin{abstract}
L'explosion démographique des sujets âgés en France est en pleine et durable expansion depuis quelques années. Si on s'intéresse au segment le plus dynamique de cette croissance, à savoir plus particulièrement les patients âgés de 80 ans et plus, par ailleurs le plus souvent polypathologiques, on identifie fréquemment soit une sous-prescription, soit une surprescription et/ou une prescription inappropriée. Trois recommandations à court et moyen terme ont été proposées : obtenir rapidement des consensus d'experts sur le thème de la prescription chez le sujet âgé, intégrer la réévaluation systématique des médicaments dans des consultations annuelles pour les 80 ans et plus, mieux coordonner les actions et la communication entre les institutions, les professionnels de santé et l'Industrie.
\end{abstract}

Mots clés : bon usage, médicament, iatrogénèse, sujet âgé

Complémentaires aux travaux d'une précédente Table Ronde de Giens ${ }^{[1]}$ portant sur des recommandations concernant l'évaluation du médicament pour cette classe d'âge, deux objectifs ont été identifiés par les membres de la Table Ronde 2004 :

1. identifier des indicateurs et des outils pour favoriser le bon usage du médicament pour cette classe d'âge ;

2. identifier des outils de communication permettant une modification des comportements et favorisant le bon usage.

\section{Constat actuel de la consommation et de l'utilisation du médicament chez le sujet très âgé en France}

\subsection{La fréquence de la polymédication}

Selon les données démographiques en France et aux EtatsUnis, la population des sujets âgés en général et des plus de 80 ans en particulier est en régulière progression démographique.

En 1999, lors du dernier recensement, les plus de 65 ans représentaient plus de 9400000 personnes en France, soit 15,7\% de la population. En 2004, cette population est de près de dix millions, soit 16,2\% de la population, selon l' INSEE (Institut National des Statistiques et des Etudes Economiques). ${ }^{[2]}$ En ce qui concerne les plus de 80 ans, ils étaient 2150000 en 1999, et 2620000 en 2004, soit une progression de près de $18 \%$.

Les données démographiques (INSEE 2003) confirment que cet accroissement (absolu et relatif) est durable, en raison de l'allongement de l'espérance de vie à la naissance chez la femme (82,9 ans) et chez l'homme (75,9 ans) en France, et de la baisse des naissances qui atteint un taux de non-renouvellement.

Dans cette population, la consommation médicamenteuse est importante. Elle représenterait $33 \%$ de la consommation médicamenteuse totale selon les données d'une étude de cohorte française. ${ }^{[3]}$ Les personnes âgées vivant en institution consomment plus de médicaments que celles vivant à domicile $(5,2$ contre 4,5 médicaments par jour). La consommation de médicaments croît avec l'âge des patients et, quel que soit l'âge considéré, les femmes en consomment plus que les hommes. Trente-sept pour cent des dépenses pharmaceutiques présentées au remboursement concernent les personnes âgées. ${ }^{[4]}$

L' URCAM (Union Régionale des Caisses d'Assurance Maladie) de Haute-Normandie a entrepris, pour le mois de mars 1999, une analyse des prescriptions de médicaments pour les personnes de plus de 65 ans. Cette étude a montré qu'en moyenne 5,5 médicaments sont délivrés par patient. Une autre étude de

\footnotetext{
* Pour la liste des participants, voir en fin d'article.
} 
l'URCAM Bretagne montrait en 2003 que les personnes de plus de 80 ans prenaient en moyenne 6,7 produits différents.

Une étude de l'URCAM Poitou-Charentes a porté sur les admissions à l'hôpital de personnes âgées de plus de 70 ans pendant 5 jours consécutifs. La consommation médicamenteuse était en moyenne de 5 médicaments par jour mais était supérieure chez les patients ayant présenté un effet indésirable. La prévalence des effets indésirables était de 12,5\%.

L'URCAM Provence-Alpes-Côte d'Azur a réalisé une étude sur la polymédication des personnes âgées de 65 ans et plus sur la base d'un questionnaire adressé à des patients sélectionnés selon des critères de remboursement de médicaments. Cette étude a montré que $28 \%$ des patients sont non observants : dans $64 \%$ des cas, la non-observance des prises est due à un oubli, tandis que dans 70,5\% des cas, elle est due à un caractère plus intentionnel (satisfaction ou insatisfaction ressentie). Par ailleurs, cette étude a montré que l'automédication est occasionnelle pour $39 \%$ des patients, et habituelle pour $25 \%$ d'entre eux.

Selon les données émanant des caisses de remboursement des spécialités en ville, (sources MSA [Mutuelle Sociale Agricole], CNAMTS [Caisse Nationale d'Assurance Maladie des Travailleurs salariés]), il apparaît que les classes les plus prescrites dans cette tranche d'âge sont, dans l'ordre, les antalgiques, les médicaments à visée cardiovasculaire, les médicaments à visée digestive et les psychotropes.

Il existe donc une vraie polymédication chez les personnes âgées en raison du cumul fréquent des pathologies à cet âge.

\subsection{L'inadéquation des prescriptions chez les personnes âgées}

Trois grandes catégories de «mésusage » sont alors identifiées :

- la sous-utilisation de médicaments utiles ;

- la surprescription de certains médicaments en l'absence d'indication patente ou de produits avec niveau de SMR (Service Médical Rendu) insuffisant ;

- la prescription inappropriée avec, par exemple, le nonrespect d'interactions connues de molécules entre elles, la redondance de traitements, des posologies ou des durées de traitement non respectées, une surveillance des effets des traitements non réalisée ou non conforme aux recommandations, et la non-réévaluation régulière du rapport bénéfice/risque et de la stratégie thérapeutique.

Aux Etats-Unis, le consensus d'un panel d'experts établit une liste utile de critères des prescriptions de médicaments inappropriés chez les plus de 65 ans. ${ }^{[5]}$
Les médicaments les plus iatrogènes (les plus fréquemment à l'origine d'hospitalisation liée à des accidents provoqués) sont, en ordre décroissant : les anticoagulants (surtout les antivitamines $\mathrm{K}[\mathrm{AVK}]$ ), les anti-inflammatoires non-stérö̈diens (AINS), les diurétiques, les inhibiteurs de l'enzyme de conversion (IEC), les $\beta$-bloquants et les anti-arythmiques, les sulfamides hypoglycémiants, les psychotropes et les médicaments à propriétés anticholinergiques.

Par rapport à ce constat, les médecins généralistes présents ont souligné la difficulté de réévaluer des prescriptions en raison de leur origine diverse (spécialistes) ou de l'attachement des malades à des médicaments de confort sensés améliorer leur qualité de vie.

\subsection{Les risques encourus}

En raison notamment de leur fragilité (altération de l'homéostasie et du métabolisme, déficit des fonctions rénales et hépatiques avec modification de la pharmacocinétique des médicaments, influence directe des pathologies associées), la iatrogénèse chez les personnes âgées de plus de 65 ans a été rapportée comme responsable de 5-10\% des hospitalisations. ${ }^{[6]}$

Les accidents médicamenteux sont deux fois plus fréquents en moyenne après 65 ans et 10-20\% de ces accidents conduisent à une hospitalisation. Une proportion importante $(>50 \%)$ de cette iatrogénèse est considérée comme évitable. ${ }^{[7]}$

Si le médicament est avant tout utile à cette population de malades de plus de 80 ans, son utilisation doit répondre, pour garantir le bon usage, à un certain nombre de précautions générales et particulières en fonction de l'état de santé de chaque malade. Les analyses des médicaments consommés dans cette population montrent d'ailleurs l'existence trop fréquente de prescriptions et d'utilisations inadéquates.

\section{Identification d'indicateurs et d'outils en faveur du bon usage}

Il est nécessaire de mieux identifier des indicateurs et des outils pour favoriser ce bon usage des médicaments chez des sujets très âgés.

Le référentiel légal du bon usage du médicament est constitué par le résumé des caractéristiques du produit (RCP) et la notice destinée au patient.

Les informations concernant le sujet âgé sont accessibles même si elles sont dispersées dans différentes rubriques du RCP (4.1 Indications thérapeutiques, 4.2 Posologie et mode d'administration, 4.4 Mises en garde spéciales et précautions particulières d'emploi, 5.2 Propriétés pharmacocinétiques) 
comme l'impose le Guideline européen (Summary of Product Characteristics [SPC] Guideline Dec. 99). ${ }^{[8]}$

Le nombre de patients très âgés inclus dans les essais de phase III reste encore insuffisant, en particulier dans les pathologies non destinées exclusivement au sujet âgé mais potentiellement utiles dans cette classe d'âge. Selon le Guideline ICH (International Conference on Harmonisation) de 1994 (ICH Topic E7-Studies in Support of Special Populations: Geriatrics ${ }^{[9]}$ ), «Les médicaments doivent être étudiés dans les différents groupes d'âges, y compris le sujet âgé, quand l'utilité est certaine. Les patients inclus dans les essais cliniques doivent être représentatifs de la population cible pour laquelle est destiné ce traitement $\gg .[9]$

L'inclusion éventuelle de la population âgée dans le « ICH$\mathrm{E}_{2} \mathrm{E}$ pharmacovigilance planning » ${ }^{[10]}$ pourrait à cet égard favoriser une optimisation de l'évaluation de la sécurité du médicament avant et surtout après l'autorisation de mise sur le marché (AMM), en tenant compte de la spécificité de cette classe d'âge.

Une évaluation spécifique des effets indésirables notifiés dans cette classe d'âge serait souhaitable.

Le bon usage du médicament et ses particularités chez le sujet âgé nécessitent aussi la prise en compte des avis de la commission de la Transparence et les recommandations de bonnes pratiques de la Haute Autorité de Santé (HAS). Il est, à cet égard, aussi utile pour le prescripteur d'être attentif au niveau du SMR des médicaments.

\section{Quelles sont les stratégies d'intervention possibles face au mésusage du médicament chez le sujet âgé de $\mathbf{8 0}$ ans et plus ?}

On peut ici distinguer les interventions individuelles, mobilisant la vigilance de chaque acteur (prescripteurs, dispensateurs), des interventions populationnelles pour lesquelles un cadre spécifique dans les actions de Santé publique sont requises pour cette classe d'âge.

\subsection{Au niveau individuel}

\subsubsection{Quand se poser systématiquement la question du bon} usage pour un patient très âgé au niveau individuel ?

Pour un malade donné, peuvent survenir les critères d'alerte suivants :

- Devant une ordonnance aberrante en nombre de produits ou en cas de risque manifeste d'interactions.

- Face à un événement intercurrent, par exemple une chute, un épisode infectieux, ou toute modification inattendue de la symptomatologie clinique et a fortiori quand ces événe- ments imposent une hospitalisation. L'hospitalisation doit résolument être un de ces moments privilégiés d'intervention sur le bon usage du médicament, avec les comptesrendus écrits d'hospitalisation à destination du médecin traitant.

- Face à un changement de situation déstabilisant de la personne âgée comme un deuil, un déménagement ou une entrée en institution, et devant tout changement climatique sévère et brutal.

- Toute nouvelle prescription est également un moment propice pour s'interroger sur les prescriptions existantes. Il ne faut pas oublier de revisiter à cette occasion toutes les prescriptions en cours, quelquefois trop mécaniquement reconduites, en réévaluant assez systématiquement leur rapport bénéfice/risque qui n'est pas immuable dans le temps.

- Les outils informatiques d'aide à la prescription peuvent être utiles, même si les signaux d'alerte doivent être hiérarchisés.

\subsubsection{Comment gérer les modifications individuelles} de prescription?

Il faut hiérarchiser les priorités thérapeutiques, tout en tenant compte des traitements symptomatiques utiles à la qualité de vie des patients. Il est par ailleurs nécessaire de modifier les prescriptions en concertation avec les autres prescripteurs, en particulier les spécialistes d'organe (ex : cardiologue). Enfin, chaque intervention doit s'attacher à obtenir, dans sa motivation, l'adhésion du patient et de son entourage (tableau I).

La mise en place rapide de réunions de consensus d'experts et l'élaboration de recommandations seraient très utiles.

\subsection{Au niveau populationnel}

\subsubsection{Quand et comment agir à un niveau populationnel,} et favoriser le bon usage et la prévention collective

de la iatrogénie ?

Une réévaluation systématique annuelle des ordonnances de prescription des patients âgés de 80 ans et plus (sur une base annuelle) pourrait être retenue dans les priorités de la mise en œuvre de la loi n ${ }^{\circ} 2004-806$ du 9 août 2004 ${ }^{[12]}$ relative à la politique de santé publique qui fixe, entre autres, comme objectifs de parvenir d'ici 5 ans à la réduction de la fréquence des événements iatrogènes d'origine médicamenteuse, survenant en ambulatoire et entrainant une hospitalisation.

Le contenu médical de ces consultations annuelles devrait être défini, de même que les critères d'alerte ou les conditions de recours à un avis spécialisé gériatrique, comme souhaité par les médecins généralistes.

Il faut donc développer une réévaluation annuelle de la prescription et l'ériger en recommandation de bonne pratique. 
Tableau I. Les questions utiles à se poser en pratique devant l'ordonnance du sujet âgé (d'après Doucet et Druesne,${ }^{[11]}$ avec autorisation)

\begin{tabular}{l} 
Les bonnes questions \\
Avant de rédiger l'ordonnance : s'informer sur le malade et ses \\
maladies \\
Quelles sont la maladie prise en charge et les pathologies associées ? \\
Quelles sont les attentes du malade? \\
Quels sont les objectifs thérapeutiques à court et moyen terme ? \\
Dispose-t-on de la liste complète de tous les médicaments actuellement \\
consommés? \\
Le malade peut-il prendre seul ses médicaments et suivre la \\
surveillance du traitement? \\
Lors de la rédaction de l'ordonnance : maîtriser le traitement \\
La prescription médicamenteuse est-elle vraiment nécessaire? \\
Quels sont les principaux paramètres pharmacocinétiques des \\
médicaments envisagés? \\
Le médicament a-t-il le meilleur « rapport bénéfice/risque »? \\
Est-il nécessaire d'adapter les posologies de ce médicament ... et des \\
autres ? \\
Les conditions d'administration sont-elles adaptées au malade? \\
La prescription est-elle suffisamment précise, claire, lisible et \\
compréhensible? \\
Tous les médecins prenant en charge le malade sont-ils informés? \\
Quelle est la durée prévue du traitement ? \\
Quels sont le projet et les modalités de surveillance ? \\
Lors des consultations de surveillance : évaluer l'efficacité et la \\
Les objectifs attendus sont-ils atteints ? \\
ont-elles changé ? \\
Un nouveau symptôme est-il en rapport avec un effet indésirable ? \\
\hline
\end{tabular}

L'éducation des malades est le deuxième volet de l'amélioration du bon usage. Une information claire, délivrée au patient, permet de s'assurer de l'obtention de son accord sur le projet thérapeutique, d'améliorer l'observance et le signalement précoce des événements iatrogènes. Il serait souhaitable, par ailleurs, de promouvoir des programmes d'éducation pour cette population des 80 ans et plus, ciblés par pathologie. Ici aussi des recommandations d'experts seraient utiles.

\section{Communiquer et modifier les comportements en faveur du bon usage}

Le constat actuel reste celui du manque de cohérence des informations disponibles. Les sources sont multiples, les communications trop ponctuelles, et les moyens mis en œuvre encore trop souvent limités.

Quelles cibles et quels moyens pour une communication efficace?

\subsection{Visant les professionnels de santé}

Il faut favoriser une information générale, coordonnée sur des messages limités, bien définis et bien séquencés dans le temps. Une évaluation de l'appropriation des messages par les cibles devrait être systématiquement effectuée.

La charte de qualité de la visite médicale (accord conventionnel passé entre Les Entreprises du Médicament [LEEM] et le Comité Economique des Produits de Santé [CEPS]), ${ }^{[13]}$ met en avant le rôle important de la délégation médicale dans la promotion du bon usage du médicament et pourrait y concourir avec une information systématique sur la classe pharmacologique des produits par exemple.

Il faudrait également favoriser une communication de proximité, dont l'impact est souvent fort, avec :

- Des réunions de groupes de professionnels dans le cadre de la formation continue et information de réseaux de médecins, pharmaciens, infirmières. Des actions pilotes sont déjà menées par la MSA en partenariat avec un groupe d'assurance privé et ont montré leur efficacité.

- L'aspect didactique des comptes-rendus d'hospitalisation pourrait s'insérer dans des actions pédagogiques.

Chaque fois que sont élaborés des documents susceptibles d'être diffusés en particulier aux professionnels de santé, une réflexion spécifique devrait avoir lieu sur la présentation de cette information et le moyen d'obtenir autant que possible une appropriation de cette information par les professionnels et les malades.

\subsection{Visant les patients et leur entourage}

- Il faut s'assurer qu'elle soit articulée avec celle des professionnels.

Un vaste programme, «La prévention de la iatrogénèse médicamenteuse évitable chez le sujet âgé », a été élaboré en partenariat avec Santé en Action, l'APNET (Association Pédagogique Nationale pour l'Enseignement de la Thérapeutique), les Conseils Ordinaux, les organismes professionnels et le LEEM, en octobre 2004. ${ }^{[13]}$ Il constitue peut-être un exemple de communication grand public sur le sujet. Il est prévu que l'impact de cette campagne sur le comportement des professionnels de santé soit évalué, règle capitale pour toute action de ce type.

- Par action directe dans la presse écrite la plus lue par cette cible (p. ex. : « Notre Temps », « Pleine Vie ») et dans l'audiovisuel.

Selon une journaliste de la Table Ronde : «Les personnes âgées reçoivent de multiples informations, le plus souvent discordantes ou contradictoires. Il serait souhaitable que les infor- 
mations relayées par les médecins, les personnels soignants, l'entourage et les médias soient concordantes. Il faut savoir que des articles sur les plus de 80 ans n'intéressent pas beaucoup les rédacteurs en chef. L'envoi régulier de fiches d'information à tous les types de relais (médias, personnel soignant, etc...) sur les nouvelles connaissances et les conseils pratiques serait certainement bienvenu. Les médecins généralistes ou gériatres, les radios, la télévision, les sites Internet et d'autres supports, tel DVD par exemple, doivent être des sources d'informations pour tous ».

\subsection{Visant une dernière cible, et non des moindres les médias eux-mêmes}

Une sensibilisation des journalistes de la presse grand public serait nécessaire, alors que les sujets portant sur les personnes âgées sont rares. Par ailleurs, il apparaît qu'aucun sujet ne soit réellement «tabou » pour ce public, et que le « continent gris » reste encore à découvrir.

Enfin, il faut tendre vers le plus de partenariat et de coordination possibles entre les Institutions, les professionnels de santé et les entreprises du médicament.

\section{Conclusion}

Trois recommandations convergentes ressortent des travaux de la Table Ronde :

1. Obtenir rapidement des consensus d'experts sur le thème de la prescription chez les sujets âgés de 80 ans et plus, ces consensus pourraient aider à la mise en oeuvre des politiques de santé en 2005.

2. Intégrer la réévaluation systématique des médicaments dans des consultations de prévention annuelles dédiées à l'octogénaire. Le contenu et la démarche de ces consultations devront se fonder sur un référentiel bien défini.

3. Coordonner les actions et la communication entre les Institutions, les Caisses, les professionnels de santé, l'Industrie et les médias pour modifier les comportements des professionnels de santé, des patients et de leur entourage, et s'assurer que la forme des messages et les actions de communication sont susceptibles d'être perçues positivement par les professionnels et les malades. Ceci pourrait se faire par l'intermédiaire d'un organisme chargé de cette coordination.

\section{Remerciements}

Les auteurs tiennent à remercier le Professeur Jean Doucet et le Docteur Marine Jeantet pour leur précieuse contribution à la finalisation de cet article.

\section{Participants}

B. Boneu (CHU Purpan, Toulouse), S. Bonin-Guillaume (CHU Timone, Marseille), S. Bonnot-Marlier (Janssen Cilag, Issy-les-Moulineaux), C.-A. Cudennec (Chiesi SA, Courbevoie), R. Bordet (Pharmacologie, Lille), J. Doucet (CHU, Rouen), L. Feldmann (Iris, Courbevoie), S. Goni (Lundbeck, Paris), M. Jeantet (CCMSA Direction Santé, Bagnolet), V. Lamarque (Baxter, Maurepas), H. Lelouet (Hôpital Henri Mondor, Créteil), S. Legrain (Hôpital Bichat, Paris), P. Maillière (Servier, Courbevoie), L. Merle (CHU Dupuytren, Limoges), M.-F. Padioleau (Paris, [journaliste]), S. Personnic (MG France, Paris), F. Piette (Hôpital Charles Foix, Ivry), P. Prévost (Direction Régionale du Service Médical, Rennes), O. Réveillaud (Bièvres, [Médecin de ville]), J.-R. Royer (Faculté de Médecine, Vandoeuvre-les-Nancy), J. Soletti (LEEM, Paris), J.-L. Vetel (Centre Hospitalier, Le Mans).

\section{Références}

1. Fouchard M, Zannad F, et les participants à la Table Ronde $\mathrm{n}^{\circ} 4$ de Giens XIX. Lecture critique, évaluation, valorisation, information sur les résultats des grands essais cliniques. Therapie 2004; 59 (3): 317-22

2. Institut National des Statistiques et des Etudes Economiques (INSEE) [online]. Available from URL: http://www.insee.fr [Accessed 2005 Jul 1]

3. Fourrier A, Dequae L, Chaslerie A, et al. Sociodemographic characteristics and polypharmacy in elderly people: data from the PAQUID Study. Post Market Surv 1993; 7: 291-8

4. Fenina A, Geffroy Y. Les comptes de la santé en 2000 : études et résultats n ${ }^{\circ} 132$, 2001. Ministère de l'Emploi et de la Solidarité. Direction de la Recherche des Etudes de l'Evaluation et des Statistiques (DREES). Paris: DREES, 2001/08

5. Fick DM, Cooper JW, Wade WE, et al. Updating the Beers criteria for potentially inappropriate medication use in older adults: results of a US consensus panel of experts. Arch Intern Med 2003; 163 (22): 2716-24

6. Gonthier R, Cathebras P, Delhomme M. Iatrogénèse médicamenteuse à l'origine d'une hospitalisation après 70 ans [abstract]. Rev Med Interne 1994; 15 Suppl. 3: 343

7. Doucet J, Jego A, Noel D, et al. Preventable and non-preventable risk factors for adverse drug events related to hospital admissions in the elderly. Clin Drug Invest 2002; 22 (6): 385-92

8. European Commission. A guideline on summary of product characteristics. 1999 Dec [online]. Available from URL: http://pharmacos.eudra.org/F2/eudralex/vol-2/C/SPCGuidRev0-Dec99.pdf [Accessed 2005 Aug 15]

9. EMEA. ICH Topic E7. Studies in support of special populations: Geriatrics. Note for guidance on studies in support of special populations: geriatrics. CPMP/ ICH/379/95. 1993 Sep [online]. Available from URL: http://www.emea.eu.int/ pdfs/human/ich/037995en.pdf [Accessed 2005 Aug 15]

10. EMEA. ICH-E $\mathrm{E}_{2} \mathrm{E}$ pharmacovigilance planning. $2004 \mathrm{Dec} 1$ [online]. Available from URL: http://www.emea.eu.int/pdfs/human/ich/571603en.pdf [Accessed 2005 Aug 15]

11. Doucet J, Druesne L. Ordonnance du sujet âgé : les questions à se poser. La Revue du Praticien-Médecine Générale. Tome 17. N 624 du 29 septembre, 2003

12. Loi $n^{\circ} 2004-806$ du 9 août 2004 relative à la politique de santé publique et liens vers les décrets d'application [online]. Available from URL: http://www.legifrance.gouv.fr/html/actualite/actualite_legislative/decrets_application/2004-806 .htm [Accessed June 2005 Jun 27]

13. Les entreprises du médicament (LEEM) [online]. Available from URL: http://www.leem.org [Accessed 2005 Aug 15]

Correspondance et offprints : Thierry Marquet, Laboratoires Lundbeck SAS, 37 avenue Pierre $\mathrm{I}^{\mathrm{er}}$ de Serbie, Paris 75008, France.

E-mail : thma@lundbeck.com 